\title{
Editorial to the Special Issue on Temporal Illusions
}

\author{
Fuat Balci $^{1 * *}$ and Argiro Vatakis ${ }^{2}$ \\ ${ }^{1}$ Department of Psychology, TMDM LAB, Koç University, Rumelifeneri Yolu 34450, Sarıer, \\ Istanbul, Turkey \\ 2Department of Psychology, Panteion University of Social and Political Sciences, \\ 136 Syggrou Ave., 17671, Athens, Greece
}

Decades-long research in interval timing has primarily focused on the psychophysical properties of this fundamental function typically in consideration of veridical timing behavior. Along the similar vein, generative models of interval timing mostly focus on the processing dynamics of the internal stop-watch in its default mode. Both of these approaches have largely overlooked the malleability of perceived time by exogenous factors such as stimulus intensity and endogenous factors such as physiological arousal. These very relations could actually help researchers better understand the representational constitution of subjective time and the processing dynamics of the internal stop-watch. This special issue covers a wide range of work on the effects of different factors on timing and time perception in humans.

Subjective time has been previously shown to dilate while observing a looming compared to receding stimulus. Sgouramani et al. tested the same effect with looming and receding biological motion and replicated the previous effects only when an auditory stimulus was congruent with the visual direction or no direction information accompanied the visual stimulus. No effect was observed with visual stimulation alone or when the accompanying auditory stimulus was incongruent with the observed direction of motion. Authors attributed the effects of the lower salience of receding stimulus given the fact that overall under reproductions.

Another stimulus that has been shown to affect perceived time is click trains. Poole et al. tested whether this effect also applied to temporal order judgments both at the behavioral level and the level of latent variables (i.e., diffusion model parameters). Prior to the temporal judgments, participants experienced either

\footnotetext{
* To whom correspondence should be addressed. E-mail: fbalci@ku.edu.tr
} 
click trains, white noise or silence. There was no effect of click trains either on temporal order judgments or diffusion parameters.

A third group of stimuli that is typically shown to affect subjective time is emotional faces. More specifically, previous work has shown that emotional faces are typically overestimated, which is typically attributed to arousal-induced alteration of clock speed. Eberhardt et al. tested the effect of emotional faces on time perception based on behavioral and event-related potentials using the temporal bisection task. Not corroborating earlier findings, they did not find any difference in temporal judgments or contingent negative variation $(\mathrm{CNV}$, which has been previously and arguably attributed to temporal accumulation) between angry and neutral faces but found a relationship between temporal categorization of ambiguous durations and modulation of CNV.

Clarke and Porubanova showed that temporal illusions can also be induced by conceptual processes. Specifically, they tested the effect of scene and object knowledge on perceived time under the prediction that semantic violations would lead to time dilation, which was confirmed based on their data.

Another set of studies investigated the role of psychological factors in the modulation of subjective time. For instance, Özoğlu and Thomaschke investigated the role of introspective accuracy on arousal-induced temporal biases and found that arousal-induced dilation of subjective time was negatively correlated with introspective accuracy. Ehret et al. also tested the relationship between time experience and psychological states. They implemented a mixture of qualitative and quantitative methods and found relations between unpleasant high time awareness and boredom, as well as pleasant low time awareness and psychological flow. Finally, Ruess et al. studied the psychological mechanism that underlies the perception of stimuli intentionally elicited as occurring earlier (i.e., intentional binding) and found that intentionality is not required for intentional binding and causality is sufficient for this effect.

Another study investigated the effect of event location on perceived time. Mungan and Kaya tested the detectability of the temporal violations in equal-accented auditory sequences and found that the detection accuracy for temporal deviations was not uniform over the sequence. The sensitivity for detecting temporal violations decreased when the violation (particularly lengthening of the gap) occurred at the final gap compared to earlier violations.

Finally, Öztel and Balcı investigated whether participants are aware of the stimulus induced biases in temporal judgments. They tested participants in a temporal bisection task using stickman walking at different speeds as the test stimulus. Replicating earlier results they showed that faster walking speed led to a leftward shift in temporal judgements and that participants are not aware of stimulus-induced biases.

This set of studies constitutes a comprehensive investigation of temporal illusions, some of which contained earlier experimental manipulations while others 
extended the scope of this research domain by using new stimulus properties and/or behavioral endpoints. Overall, results reported in this special issue point at the malleability of time perception based on a number of external stimulus features and psychological states. As visual illusions have significantly contributed to the understanding of the visual perception and of how the visual system operates, we think that temporal illusions would be informative grounds over which our understanding of interval timing and internal stop-watch can be built. 